\title{
Probing $\alpha$-helical and $\beta$-sheet Structures of Peptides at Solid/Liquid Interfaces with SFG
}

\author{
Xiaoyun Chen, Jie Wang, Jason J. Sniadecki, Mark A. Even, Zhan Chen* \\ Department of Chemistry, University of Michigan, Ann Arbor, MI 48109
}

\section{Materials}

Tachyplesin I was purchased from Becham (King of Prussia, PA). In the literature it is found to exert its bactericidal activity by permeating bacterial membranes. One side of tachyplesin I is crowded with a cluster of hydrophobic bulky side chains while the other side is occupied by a cluster of positively charged side chains. This renders tachyplesin I very amphiphilic. Many studies employing mutated tachyplesin I have been carried out in an effort to understand its structure-function relation but its antimicrobial mechanism still remains elusive. ${ }^{1}$ MSI594 was synthesized by the Genaera Corporation and provided by the Ramamoorthy Group at the University of Michigan. MSI594 is an analog of magainin I. Due to the dichotomy in the side chain hydrophobicities, peptides in the magainin family are amphiphilic and are found to exert their antimicrobial activity by permeating the bacterial membrane, though the exact mechanism reamins an issue of debate. ${ }^{2}$ In this paper, both peptides were dissolved in phosphate buffer saline (PBS, pH 7.4) and diluted to a final concentration of $100 \mathrm{ug} / \mathrm{ml}$ for adsorption. The deionized water $(18.2 \mathrm{M} \Omega-\mathrm{cm})$ was obtained from a Millipore ultrapure water system. Polystyrene (Mw 250,000) was 
purchased from Scientific Polymer Products and DTT was from Sigma and both were used as received. Polymer films were prepared by spin coating. A spin coater from Specialty Coating Systems was used to spin coat 2\% PS/toluene solution at $2500 \mathrm{rpm}$ on calcium fluoride prism (ISP) to make PS films.

\section{Peptide Adsorption and Experiment on DTT Treatment}

A total reflection geometry employing a $\mathrm{CaF}_{2}$ prism coated with PS was adopted for all SFG experiments. The PS film on a $\mathrm{CaF}_{2}$ prism was immersed in PBS first. Then the peptide solution was injected into the buffer solution to a concentration of $100 \mathrm{ug} / \mathrm{ml}$ and allowed to adsorb to the PS surface. Spectra taken during the initial period underwent some change in both spectral feature and intensity due to the diffusion and adsorption process. Normally after about 15 minutes the spectra became stable and no detectable time-dependent change was observed. All the spectra reported here are averaged results of the stable spectra.

For the in-situ DTT experiment, a concentrated DTT solution was injected into the tachyplesin I solution already in contact with PS film. The final concentration of DTT is $10 \mathrm{mM}$, which is about 113 times the molar concentration of the disulfide bonds. By

setting the IR beam frequency at $1688 \mathrm{~cm}^{-1}$ and monitor the intensity of SFG signal as a function of time, we could observe a gradual signal decrease that occurred on a time scale of a few minutes (result not shown here). For the ex-situ DTT experiment, DTT was added to $100 \mathrm{ug} / \mathrm{ml}$ tachyplesin I to a final concentration of $10 \mathrm{mM}$. Then the mixture solution of DTT and tachyplesin I was allowed to react for 5 minutes and then brought into contact with PS film and a SFG spectrum was taken. Almost identical spectra were 
obtained for the in situ and ex situ treated tachyplesin I at the PS/tachyplesin I solution interface.

\section{ATR-FTIR}

ATR-FTIR was also used as a complementary technique to further confirm the conformation of peptides at the interfaces deduced by SFG studies. ATR-FTIR spectra were collected using a Nicolet-550 spectrometer. A thin layer of polystyrene (PS) film was deposited onto the $\mathrm{ZnSe}$ total reflection element by solvent casting with $0.1 \%$ PS solution and then dried with $\mathrm{N}_{2}$. The film was then immersed in PBS buffer overnight before the peptide adsorption experiment. To keep consistent with our SFG experiment, $100 \mathrm{ug} / \mathrm{ml}$ peptide solution was used.

\section{Discussion about SFG Experiment, Spectral Interpretation, and Peak Assignment:}

Total Reflection Geometry: As mentioned in the paper, we adopted a total reflection experimental geometry to collect SFG spectra presented in this communication. The Fresnel coefficient may be very sensitive to changes of refractive index. The wavelength dependent refractive index can change near a resonance and may substantially distort an SFG spectrum. However, we believe that they will not greatly affect our SFG spectra here. We have used other experimental geometries, e.g., a window geometry, in which the incident laser beam angles are much smaller than the critical angles for total reflection, to evaluate the possible spectral distortion of the total reflection geometry. The spectral features collected from different experimental geometries are very similar.

Interfacial Peptides: We believe that the SFG signal is from the tightly adsorbed interfacial peptide molecules. To prove this we first collected SFG spectra from the PS/tachyplesin I solution interface. We then removed PS from the tachyplesin I solution, 
rinsed the PS surface with buffer, and contacted the PS with PBS buffer again. SFG spectra collected from the PS/buffer interface were then collected. The spectra collected from the initial PS/tachyplesin I solution interface are identical to those collected from the PS/buffer interface, indicating that the SFG signal from the PS/tachyplesin I solution interface is mainly contributed by the interfacial tightly adsorbed tachyplesin I layer. Similar experiments with MSI594 were also performed and no change in spectra collected before and after rinsing was observed either.

Spectral Fitting: To fit the spectra, the following equation was used: $I(\omega) \propto\left|\chi_{m r}+\sum_{q} \frac{A_{q}}{\omega-\omega_{q}+i \Gamma_{q}}\right|^{2}$

where $\chi_{\mathrm{nr}}$ arises from the nonresonant background contribution, and $\mathrm{A}_{\mathrm{q}}, \omega_{\mathrm{q}}$, and $\Gamma_{\mathrm{q}}$ are the strength, resonant frequency, and damping coefficient of the vibrational mode q. Therefore $\mathrm{A}_{\mathrm{q}}, \omega_{\mathrm{q}}$, and $\Gamma_{\mathrm{q}}$ can be obtained by fitting the SFG spectra.

For all the fitting results, some peaks in the lower wavenumber range $\left(1500-1610 \mathrm{~cm}^{-1}\right)$ are needed to obtain a satisfactory fitting. The origin of those bands is not clear yet. They also appear in the PS/PBS background with a varied intensity in different experiments. We think they may be from the C-C stretching mode of the PS. Further study to clarify the origin of these peaks is under way.

The fact that SFG component peaks do not seem to add up to the total spectral feature is due to the fact that SFG is a coherent process and SFG signals from each contributing component do not simply linearly add up. Instead they will interfere constructively or destructively, depending on their phases. In addition the intensity of SFG signal is proportional to the square of the effective susceptibility. Another factor that may skew 
the spectral feature is the non-resonant background. A nonresonant background was found for all the SFG spectra shown in this paper. For example, because of the interference between the nonresonant background and the vibrational resonant peak, the peak center of the SFG spectrum collected from the PS/MSI594 solution interface exibit an apparent shift.

We want to point out that the entire fitting process is carried out with an intention to identify $\alpha$-helical and $\beta$-sheet secondary structures. Spectral assignments for tachyplesin I in the range lower than $1670 \mathrm{~cm}^{-1}$ are less straightforward than the assignment for the $1688 \mathrm{~cm}^{-1}$ peak due to the extensive overlapping of several possible contributing bands from turns, random structures, $\beta$-sheet, etc. We referenced ATR-FTIR fitting result when fitting the SFG spectra. Further SFG study in the amide I range and polarization mapping method may give a more conclusive result. ${ }^{3}$

The tentative assignment scheme for tachyplesin I at the PS/solution interface is : 1688 $\mathrm{cm}^{-1}-\mathrm{B}_{1} / \mathrm{B}_{3}$ mode of $\beta$-sheets; $1664 \mathrm{~cm}^{-1}$ - turns/random structures; $1645 \mathrm{~cm}^{-1}$-random structure/ $\mathrm{B}_{2}$ mode of $\beta$-sheets; $1605 \mathrm{~cm}^{-1}, 1574 \mathrm{~cm}^{-1}$ - unknown origin, possibly from PS.

In contrast spectral assignments are much more straightforward for MSI594 adsorbed at the PS/solution interface. The dominant peak is at $1650 \mathrm{~cm}^{-}$. Two other peaks of much lower strength at $1615 \mathrm{~cm}^{-1}$ and $1573 \mathrm{~cm}^{-1}$ (unknown origin, possibly from PS) are also detected from the fitting.

\section{Reference}

1 (a) Matsuzaki, K.; Nakayama, M.; Fukui, M.; Otaka, A.; Funakoshi, S.; Fujii, N.; Bessho, K.; Miyajima, K. Biochemistry 1993, 32, 11704-11710. (b) Park N G; Lee S; 
Oishi O; Aoyagi H; Iwanaga S; Yamashita S; Ohno M Biochemistry, 1992, 31, 1224112247.

2. (a) Matsuzaki, K. Biochimica Et Biophysica Acta 1998, 1376, 391-400. (b) Matsuzaki, K. Biochimica Et Biophysica Acta 1999, 1462, 1-10.

3. Wang, J.; Clarke, M. L.; Chen, Z. Analytical Chemistry 2004, 76, 2159-2167. 